Supporting Information

\title{
Tris(phosphaalkyne)tungsten complexes
}

\author{
Daniel W. N. Wilson, Nicholas H. Rees and Jose M. Goicoechea* \\ Department of Chemistry, University of Oxford, Chemistry Research Laboratory, 12 \\ Mansfield Road, Oxford, OX1 3TA, U.K. \\ E-mail: jose.goicoechea@chem.ox.ac.uk
}

\section{Contents}

1. Characterisation Data

2. Single crystal X-ray diffraction data

3. Computational Data

4. References 


\section{Characterisation Data}

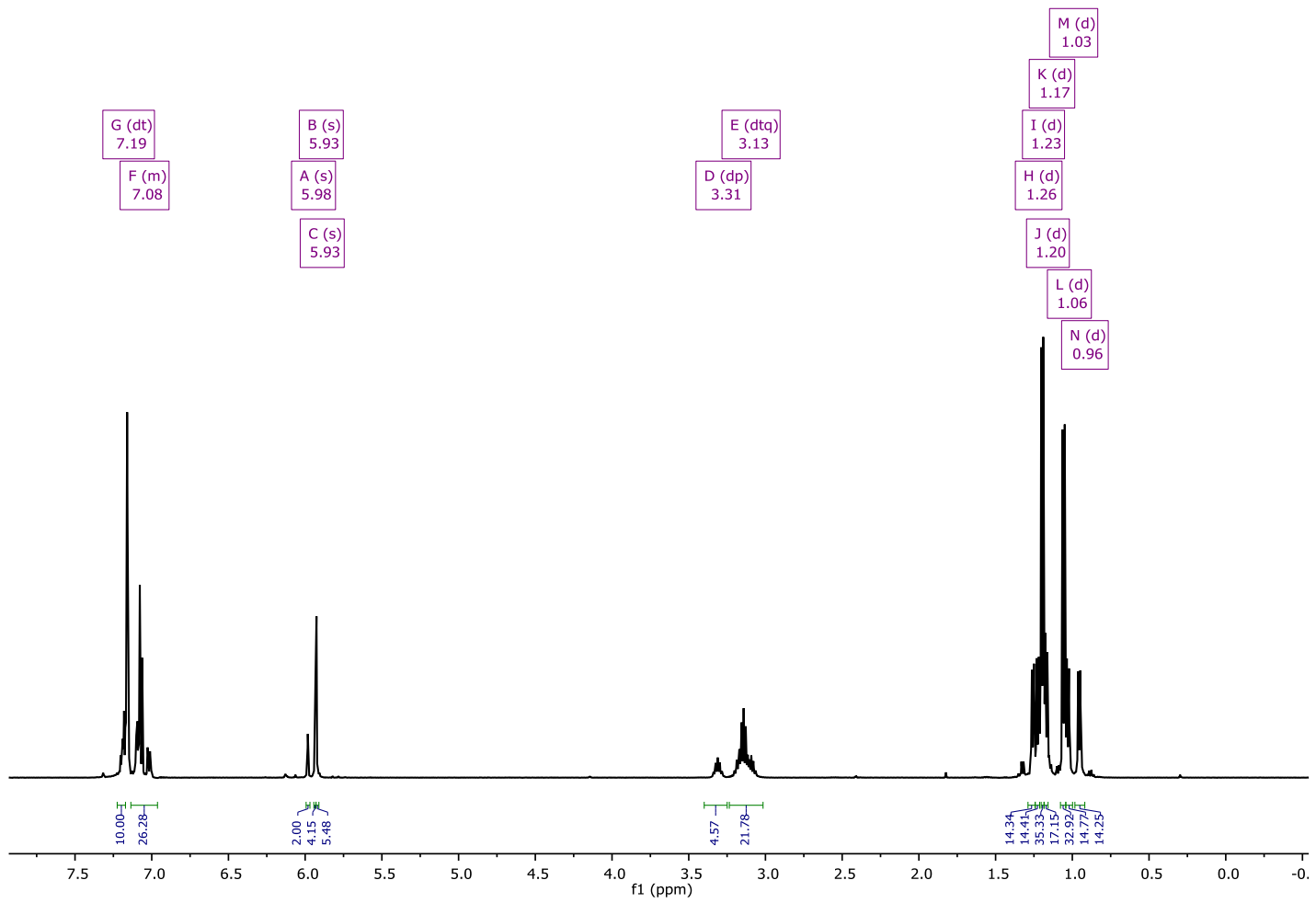

Figure S1: Room temperature ${ }^{1} \mathrm{H}$ spectrum of 2 in $\mathrm{C}_{6} \mathrm{D}_{6}$.

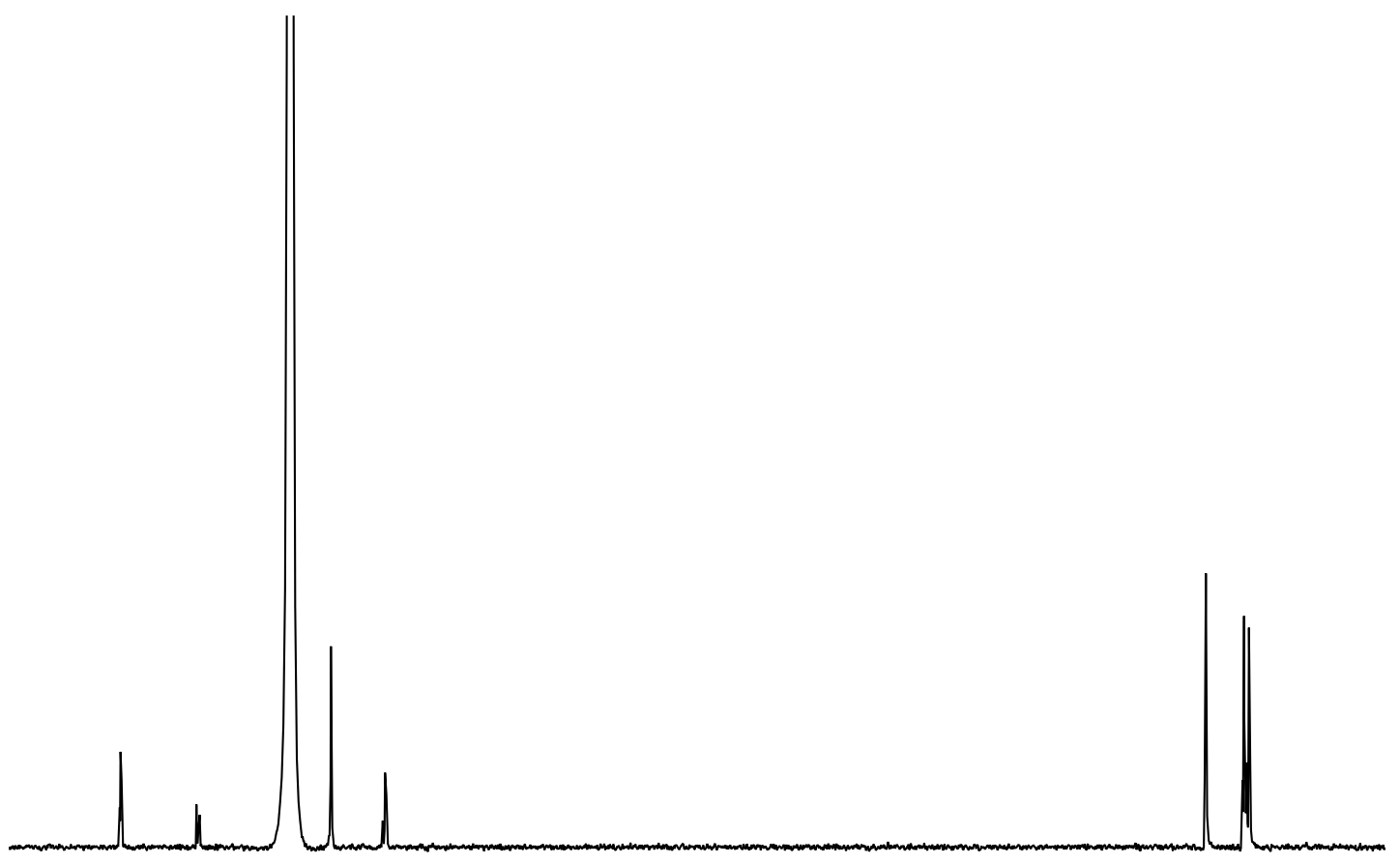

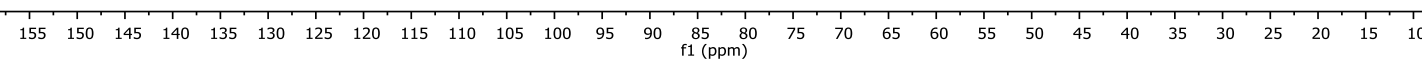

Figure S2: Room temperature ${ }^{13} \mathrm{C}\left\{{ }^{1} \mathrm{H}\right\}$ spectrum of 2 in $\mathrm{C}_{6} \mathrm{D}_{6}$. 


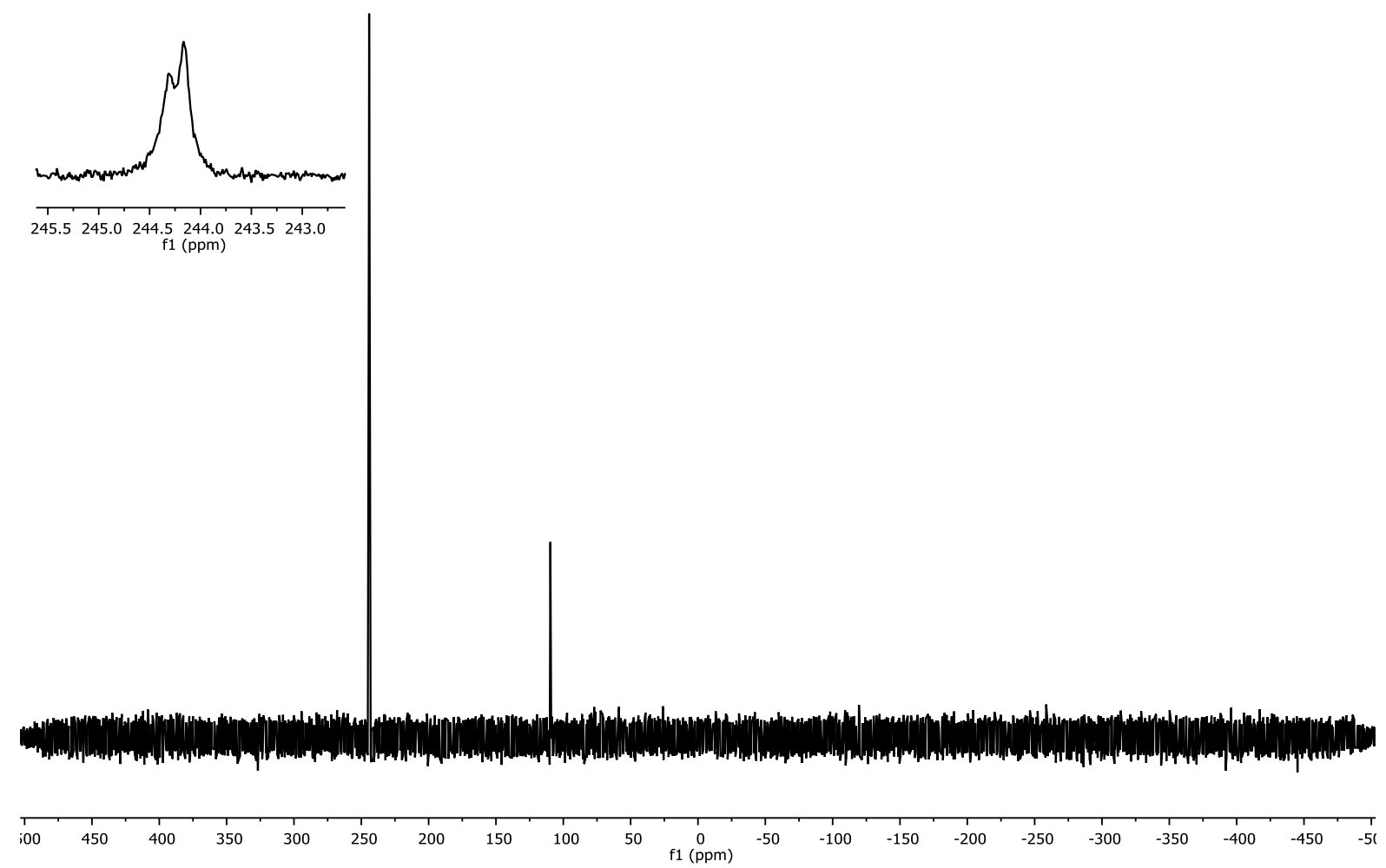

Figure S3: Room temperature ${ }^{31} \mathrm{P}\left\{{ }^{1} \mathrm{H}\right\}$ spectrum of 2 in $\mathrm{C}_{6} \mathrm{D}_{6}$.

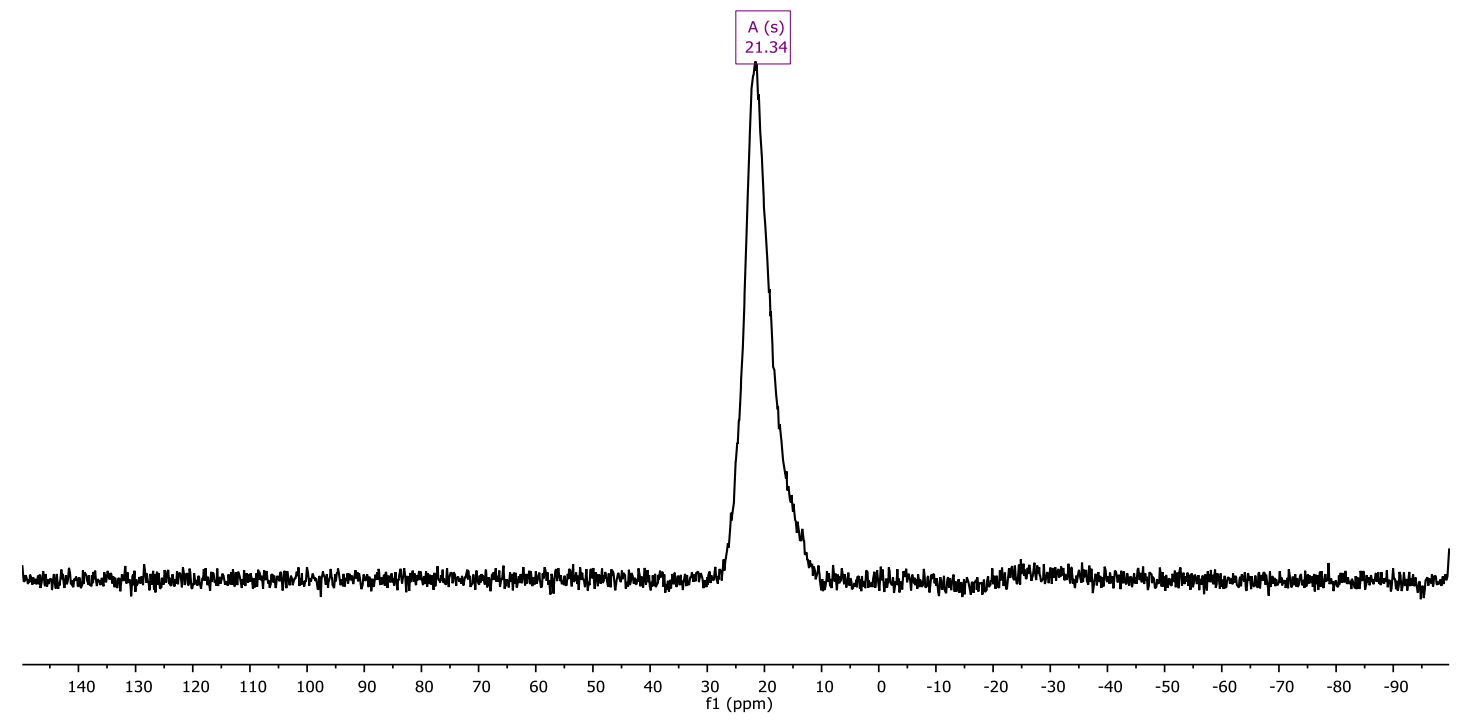

Figure S4: Room temperature ${ }^{11} \mathrm{~B}\left\{{ }^{1} \mathrm{H}\right\}$ spectrum of $\mathbf{2}$ in $\mathrm{C}_{6} \mathrm{D}_{6}$. 


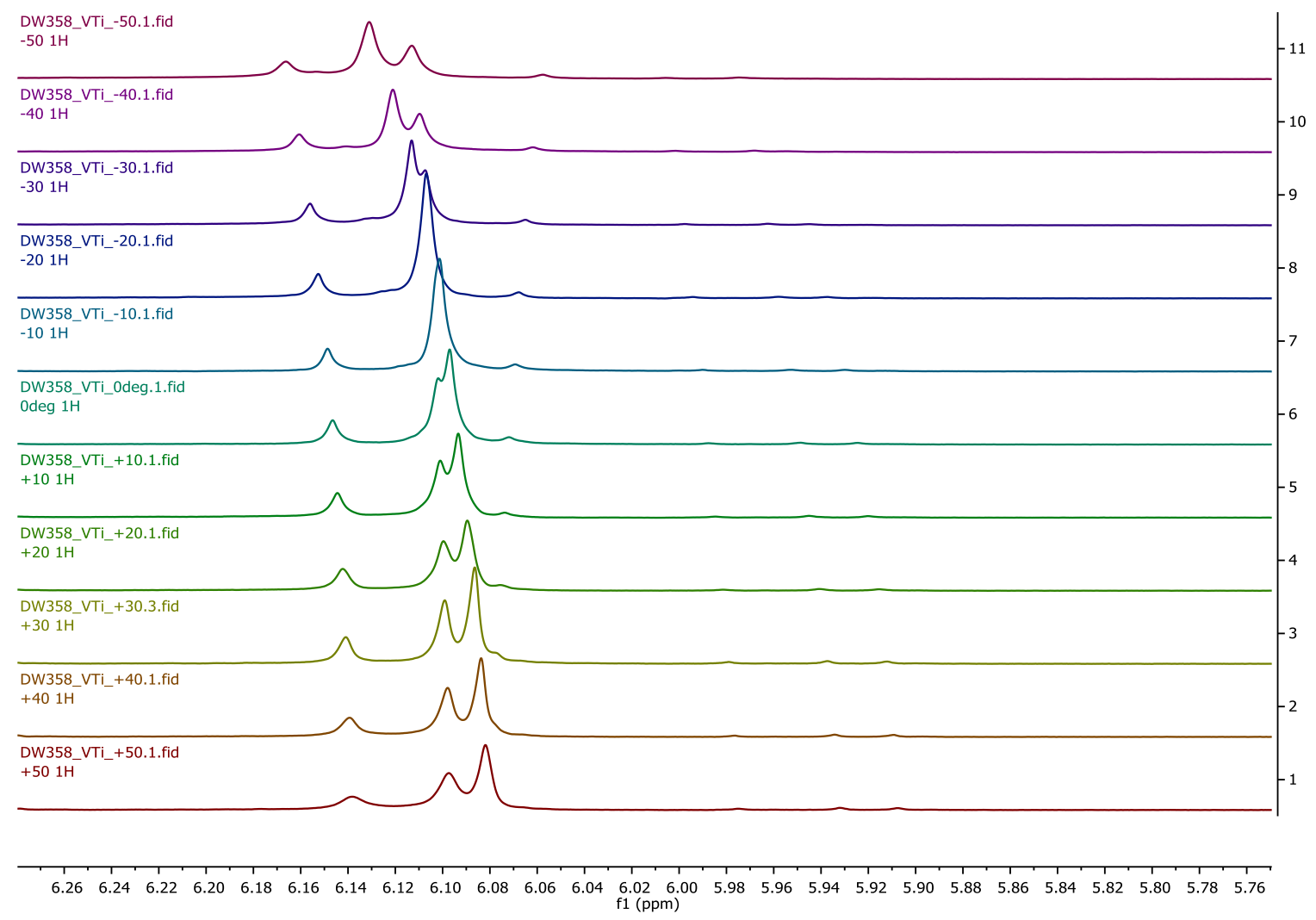

Figure S5: Variable temperature $\left(+50\right.$ to $\left.-50^{\circ}\right){ }^{1} \mathrm{H}$ NMR spectrum of 2 in $\mathrm{d}^{8}$-toluene, boryl backbone region highlighted. 


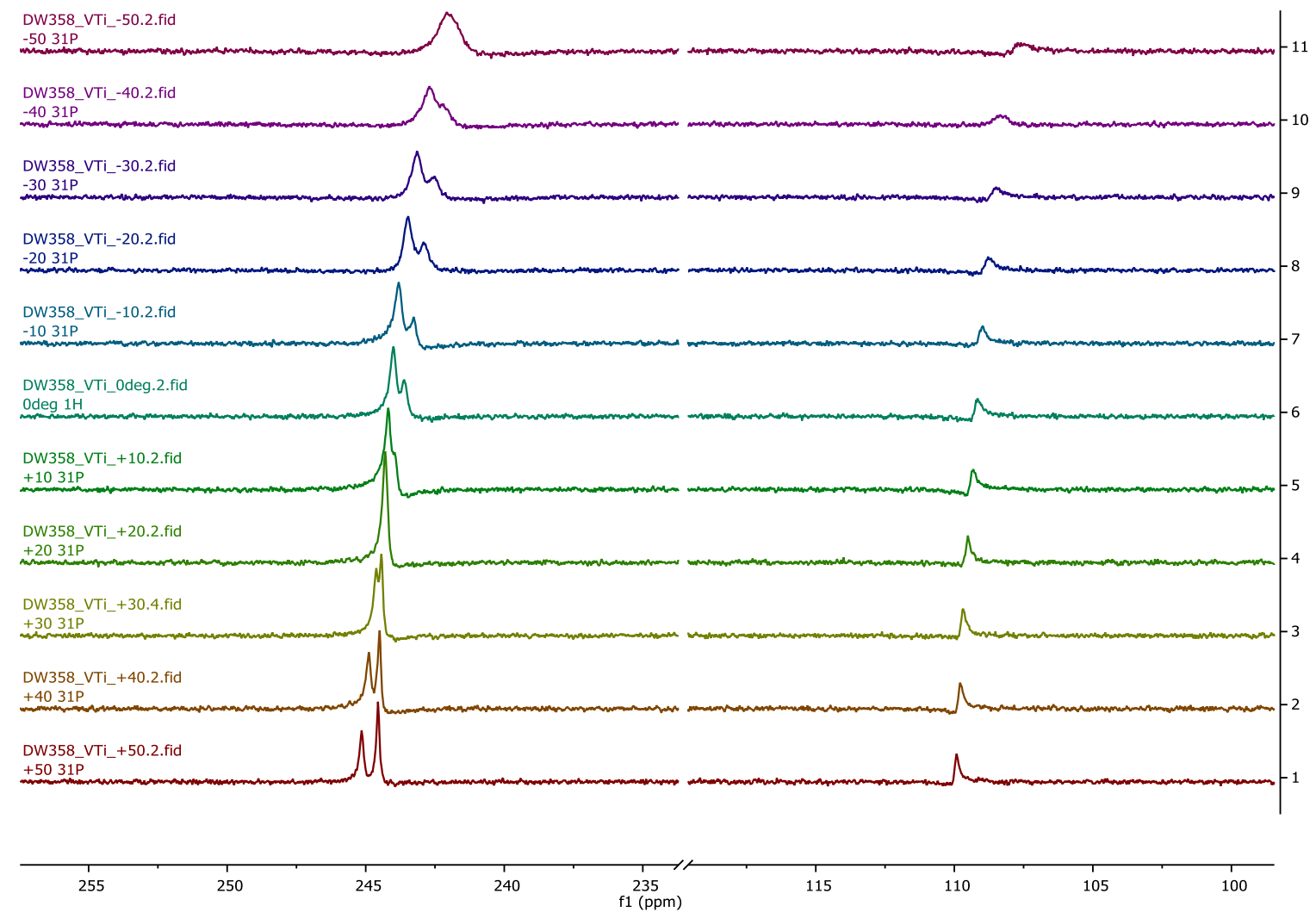

Figure S6: Variable temperature $\left(+50\right.$ to $\left.-50^{\circ}\right){ }^{31} \mathrm{P}$ NMR spectra of $\mathbf{2}$ in $\mathrm{d}^{8}$-tol.

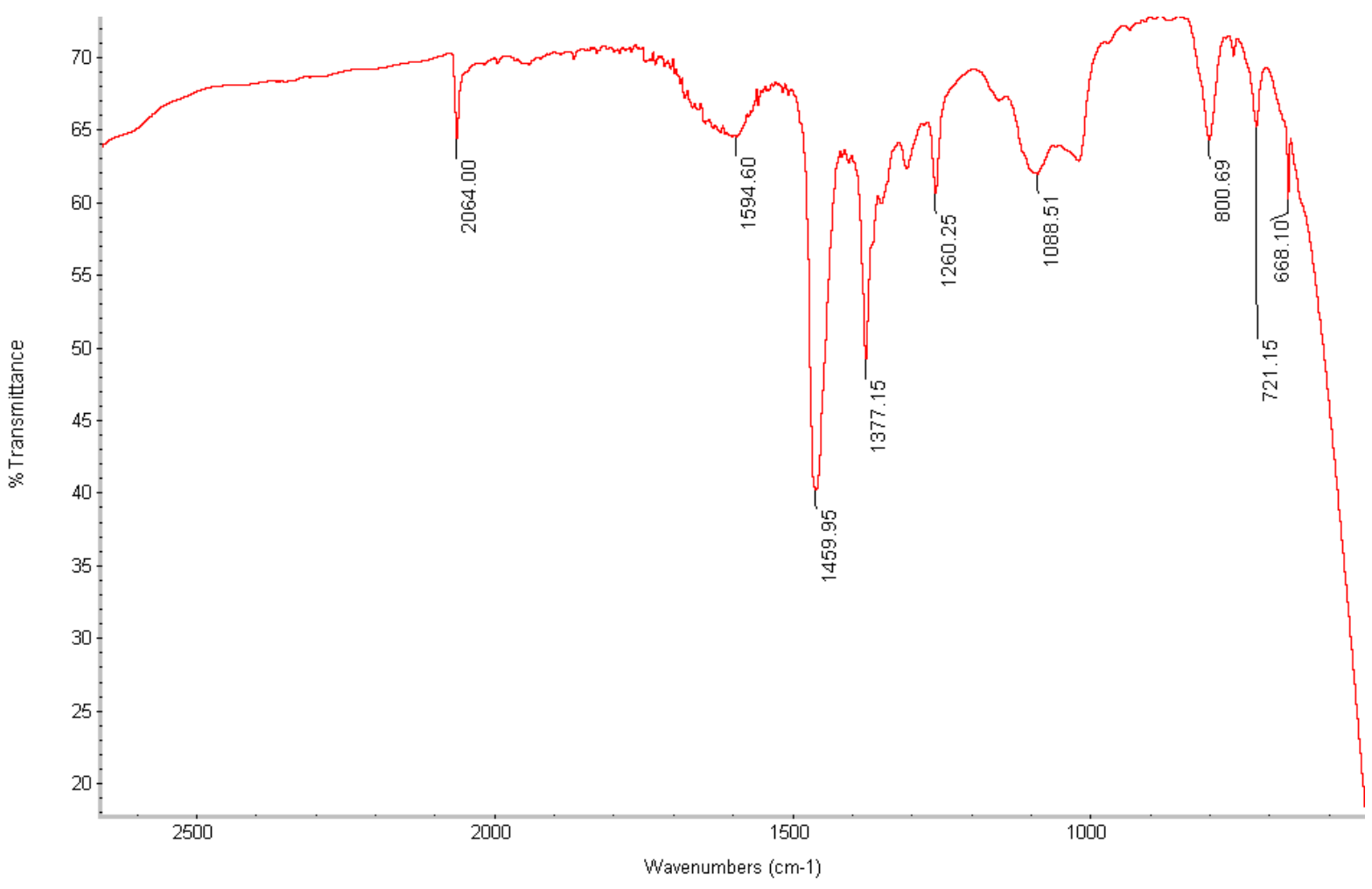

Figure S7: Solid state IR spectrum of 2. 


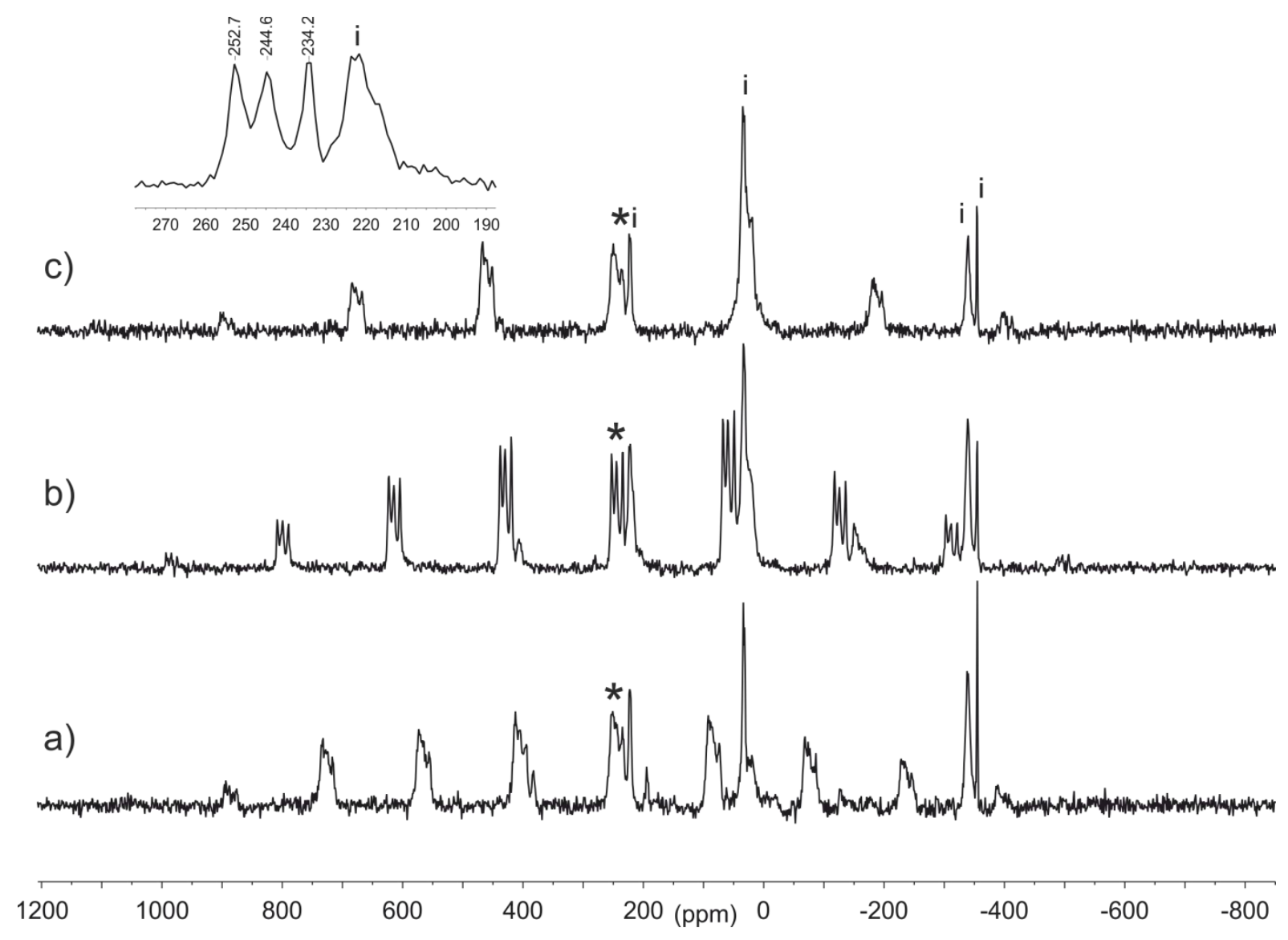

Figure S8: ${ }^{31} \mathrm{P}\left\{{ }^{1} \mathrm{H}\right\}$ CPMAS spectra of 2; a) spinning speed $26 \mathrm{kHz}$, b) spinning speed 28 $\mathrm{kHz}, \mathrm{c}$ ) spinning speed $35 \mathrm{kHz}$, * indicates isotropic peak for $\mathrm{P} \equiv \mathrm{N}$ resonances (see inset expansion) and i indicates impurity peaks. 


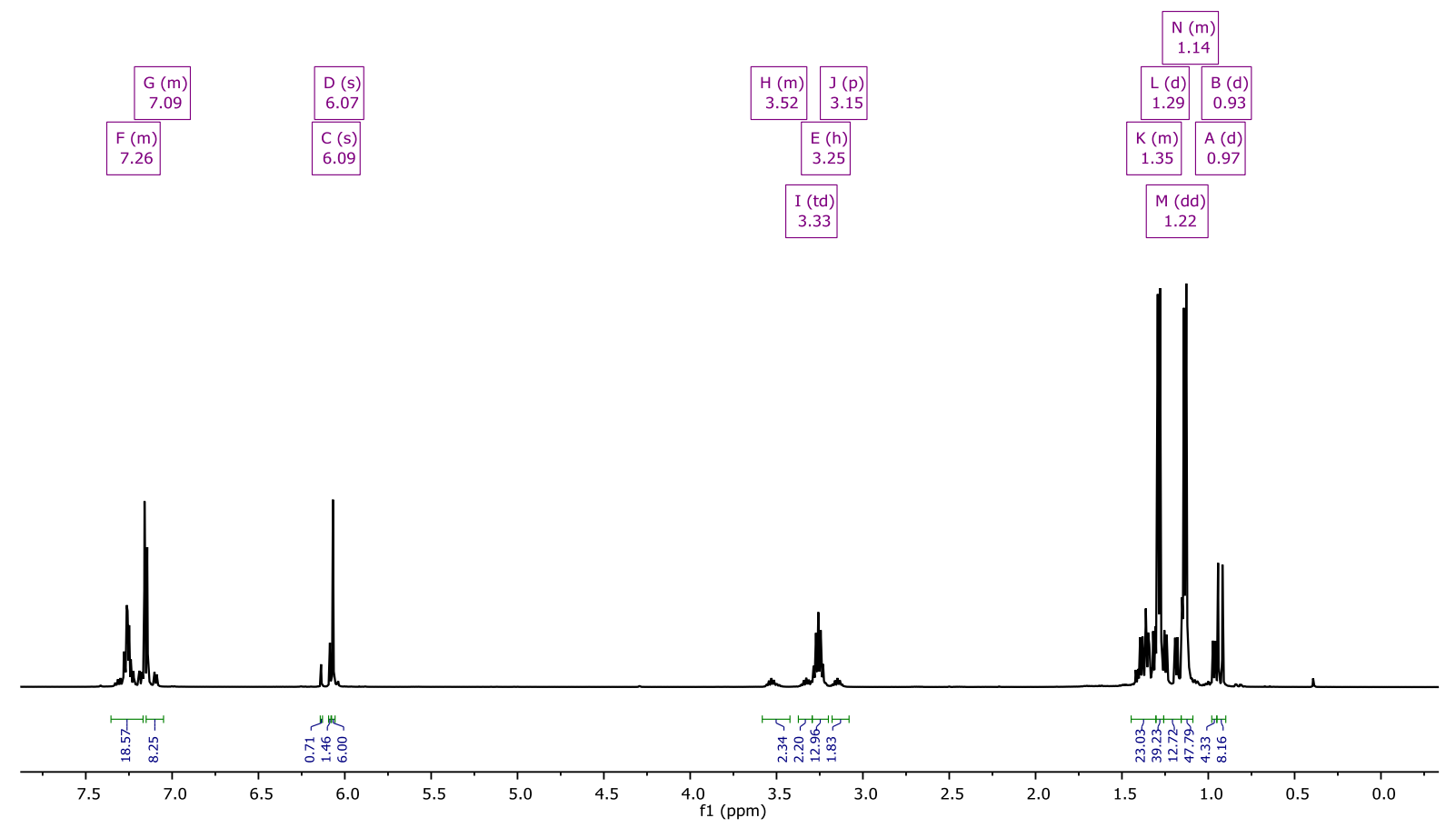

Figure S9: Room temperature ${ }^{1} \mathrm{H}$ spectrum of 3 in $\mathrm{C}_{6} \mathrm{D}_{6}$.

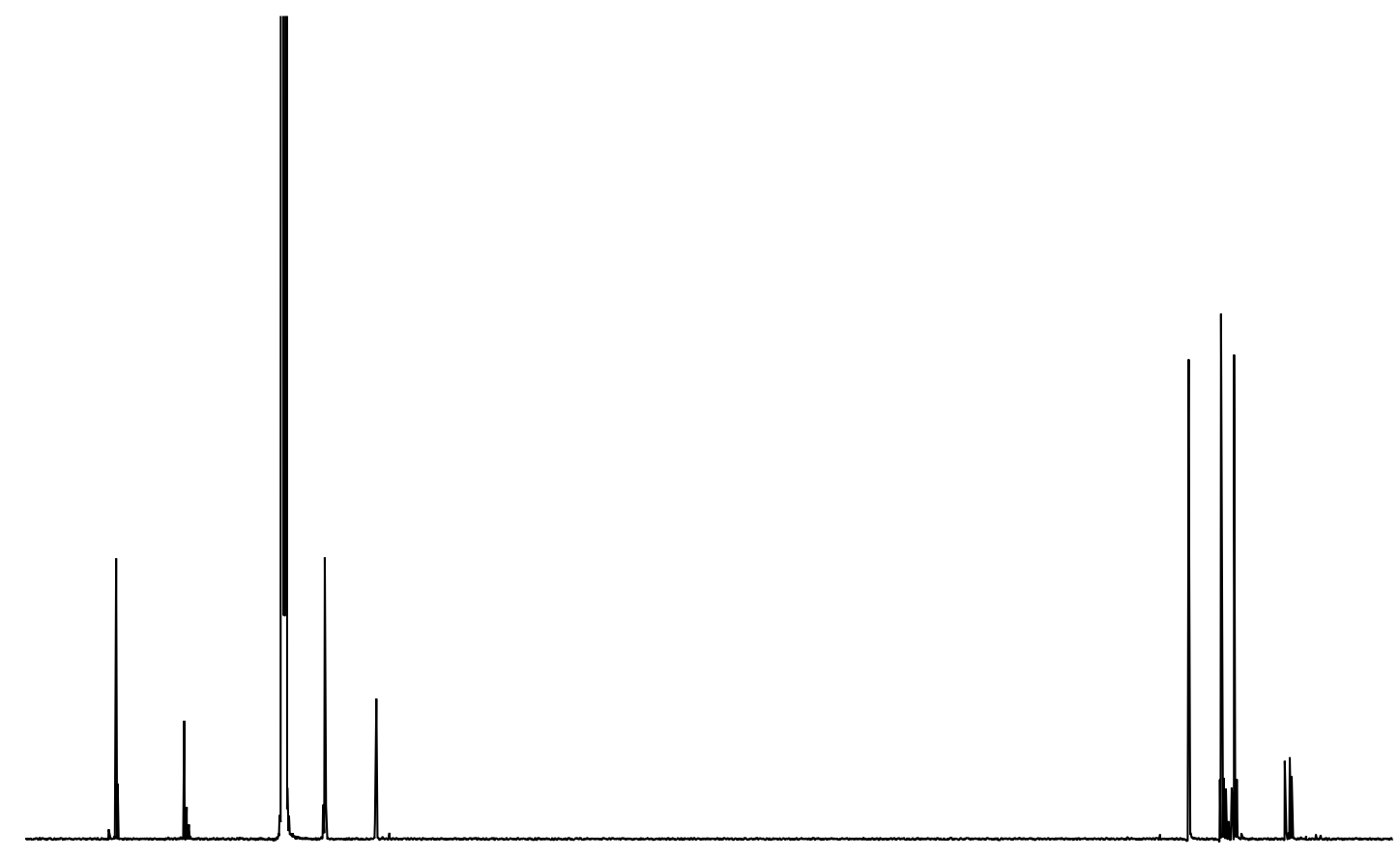

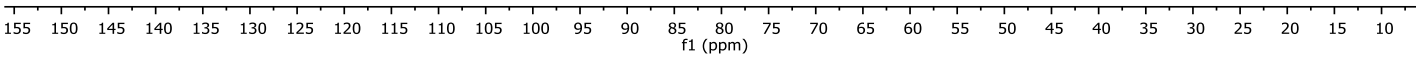

Figure S10: Room temperature ${ }^{13} \mathrm{C}\left\{{ }^{1} \mathrm{H}\right\}$ spectrum of 3 in $\mathrm{C}_{6} \mathrm{D}_{6}$. 


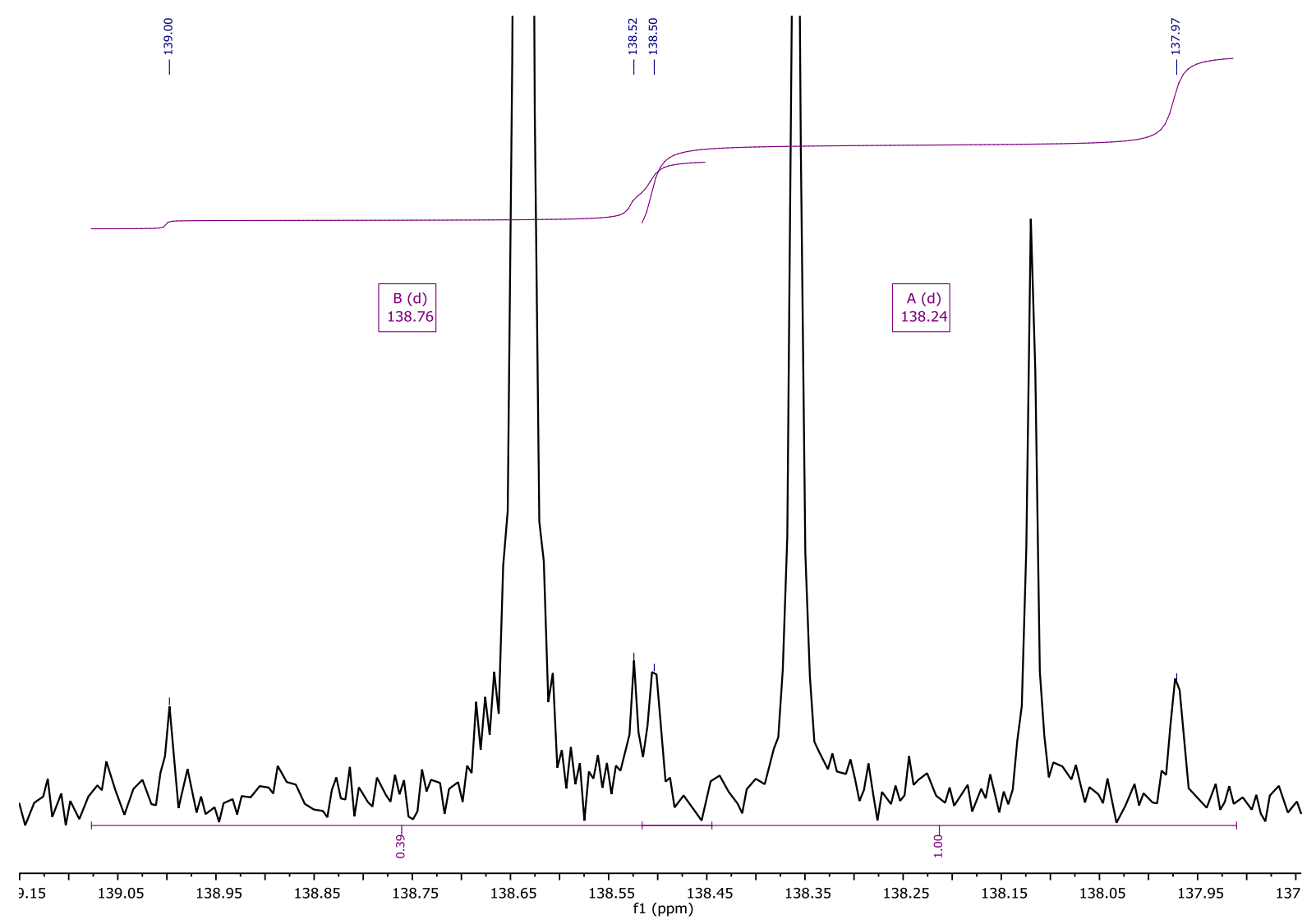

Figure S11: Enhancement of region showing $C \mathrm{P}$ doublets in the ${ }^{13} \mathrm{C}\left\{{ }^{1} \mathrm{H}\right\}$ spectrum of 3 .

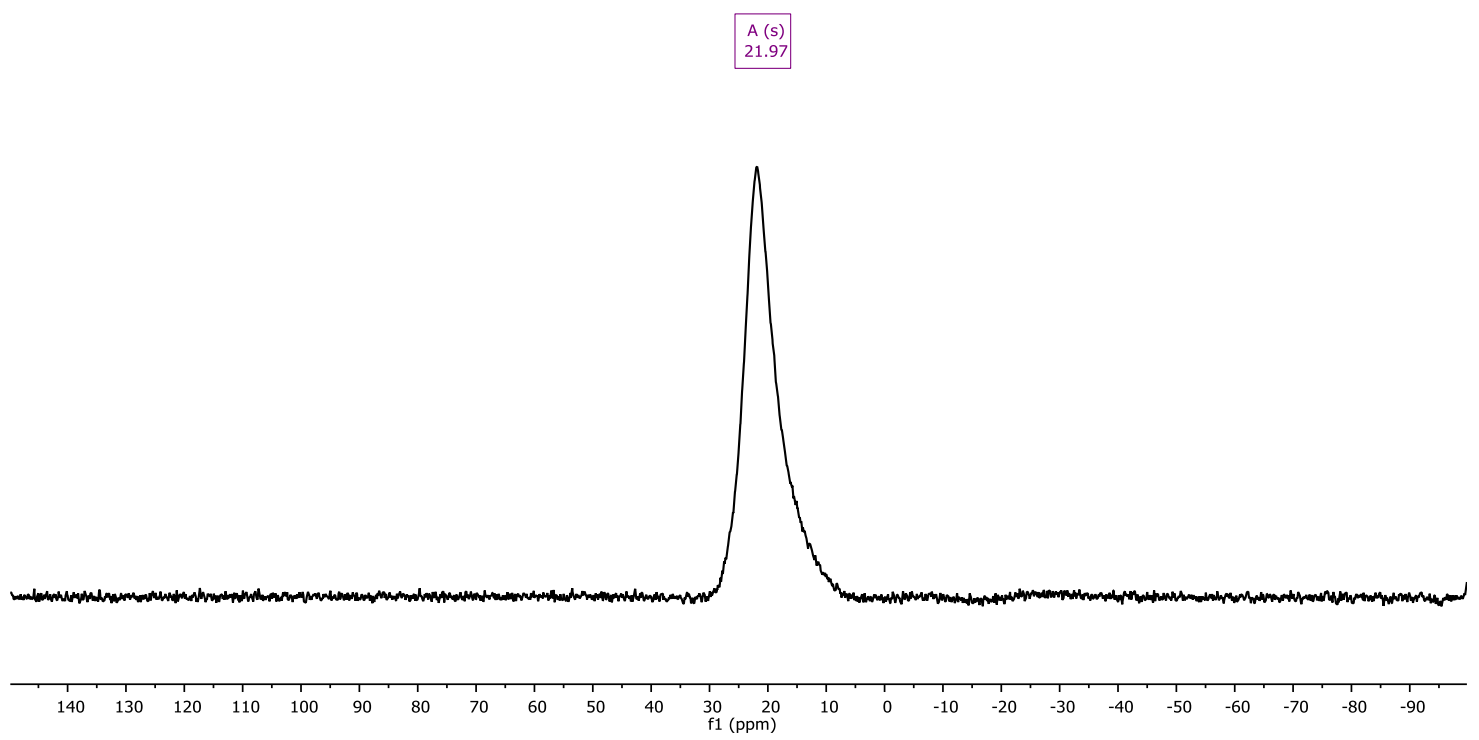

Figure S12: Room temperature ${ }^{11} \mathrm{~B}\left\{{ }^{1} \mathrm{H}\right\}$ spectrum of $\mathbf{3}$ in $\mathrm{C}_{6} \mathrm{D}_{6}$. 


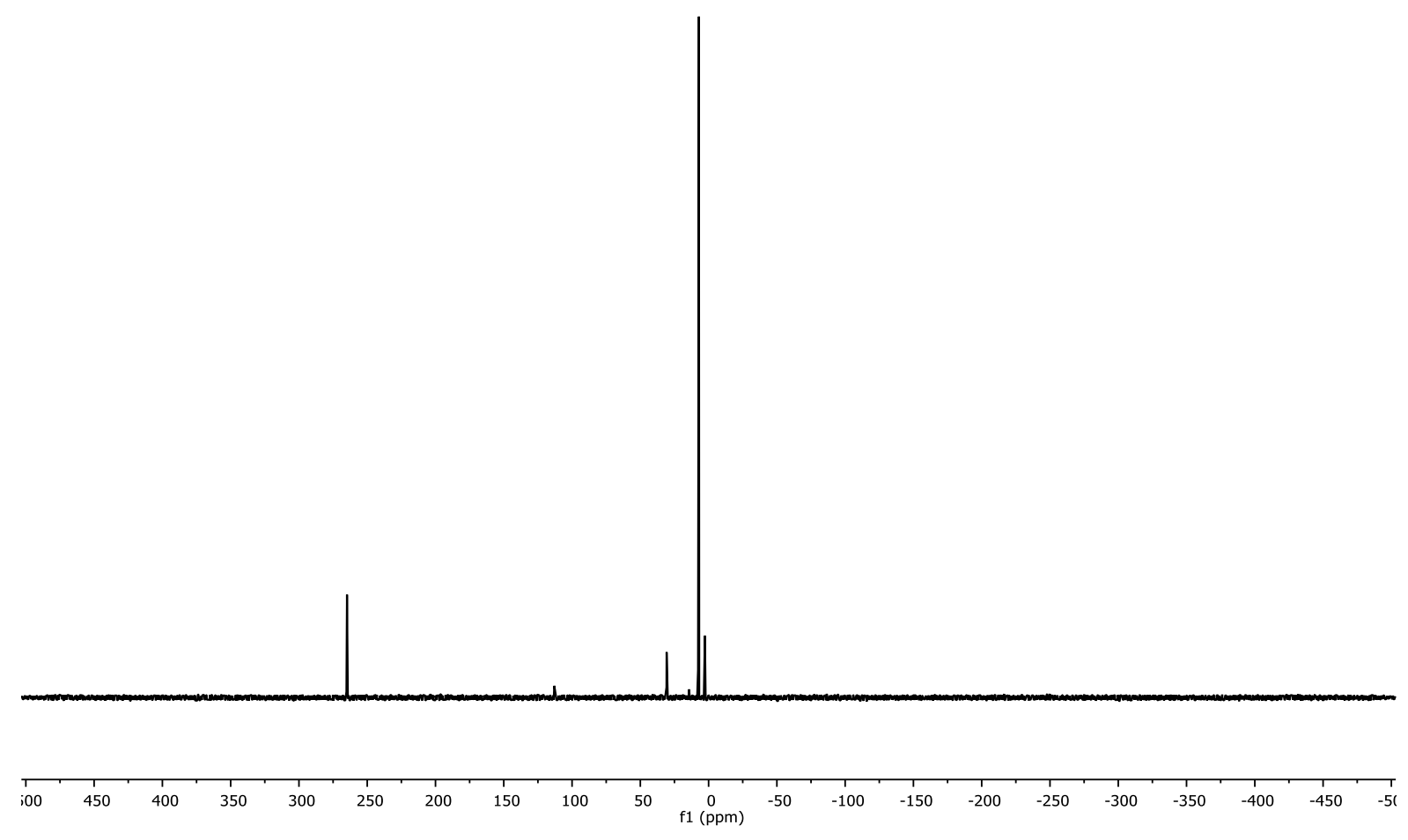

Figure S13: Room temperature ${ }^{31} \mathrm{P}\left\{{ }^{1} \mathrm{H}\right\}$ spectrum of $\mathbf{3}$ in $\mathrm{C}_{6} \mathrm{D}_{6}$.

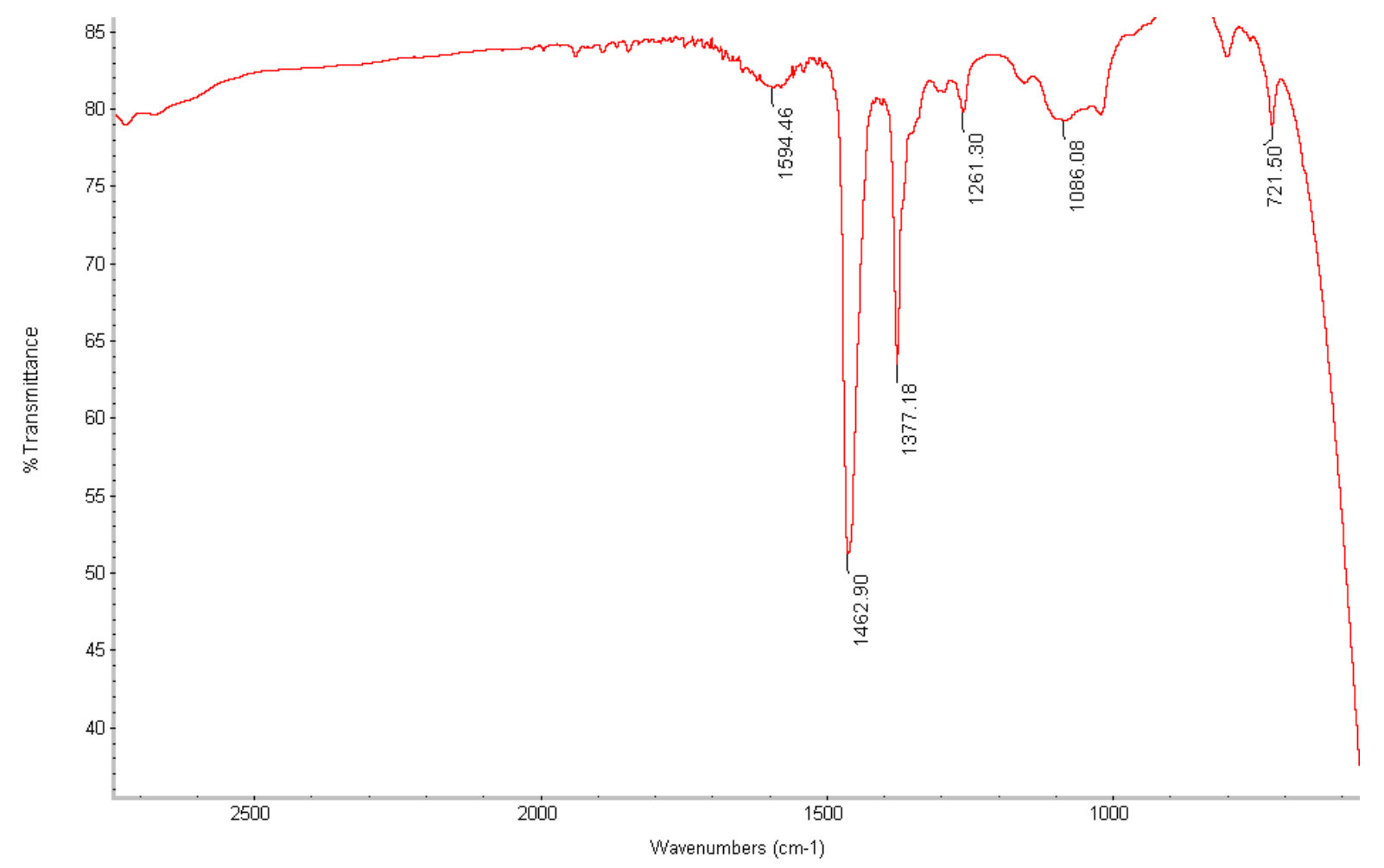

Figure S14: Solid state IR spectrum of 3. 
e)

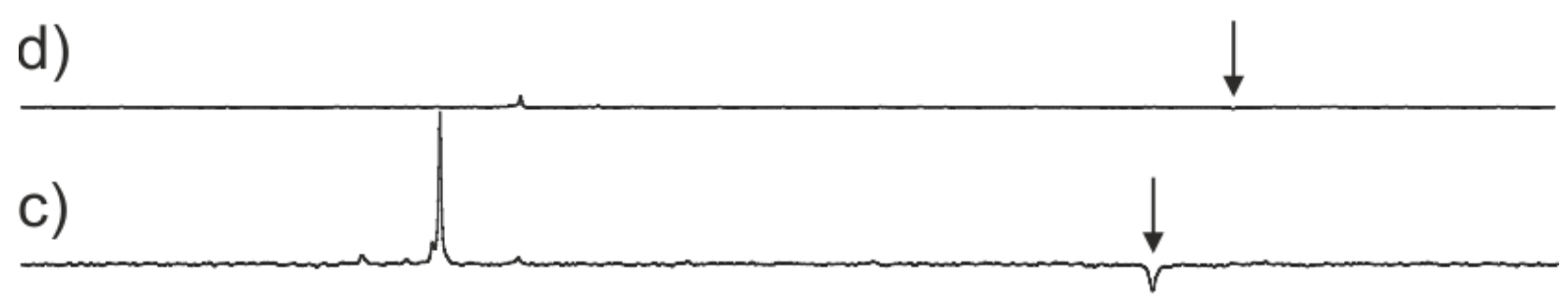

b)

a)
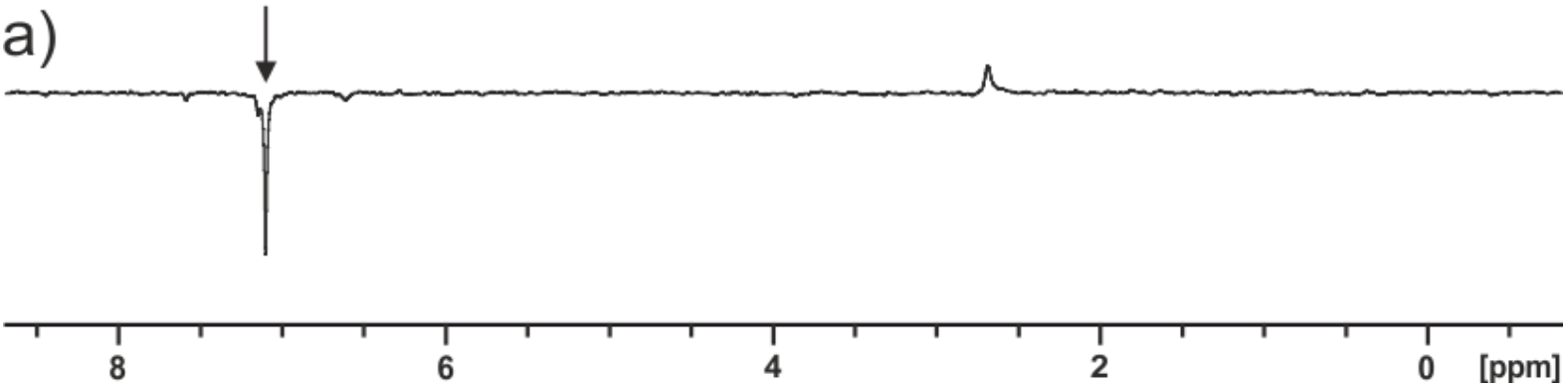

Figure S15: ${ }^{31} \mathrm{P}\left\{{ }^{1} \mathrm{H}\right\}$ selective inversion 1D-EXSY spectra of $\mathbf{3}$ for the $\mathrm{PMe}_{3}$ peaks of both isomers; a) major isomer peak inverted at mixing time $0 \mathrm{~s}$, b) major isomer peak inverted after 3s mixing time, c) minor isomer pear inverted at mixing time $0 \mathrm{~s}$, d) minor isomer peak inverted after 3 s mixing time, e) ${ }^{31} \mathrm{P}\left\{{ }^{1} \mathrm{H}\right\}$ spectrum of same region (arrow indicates inverted peak) 


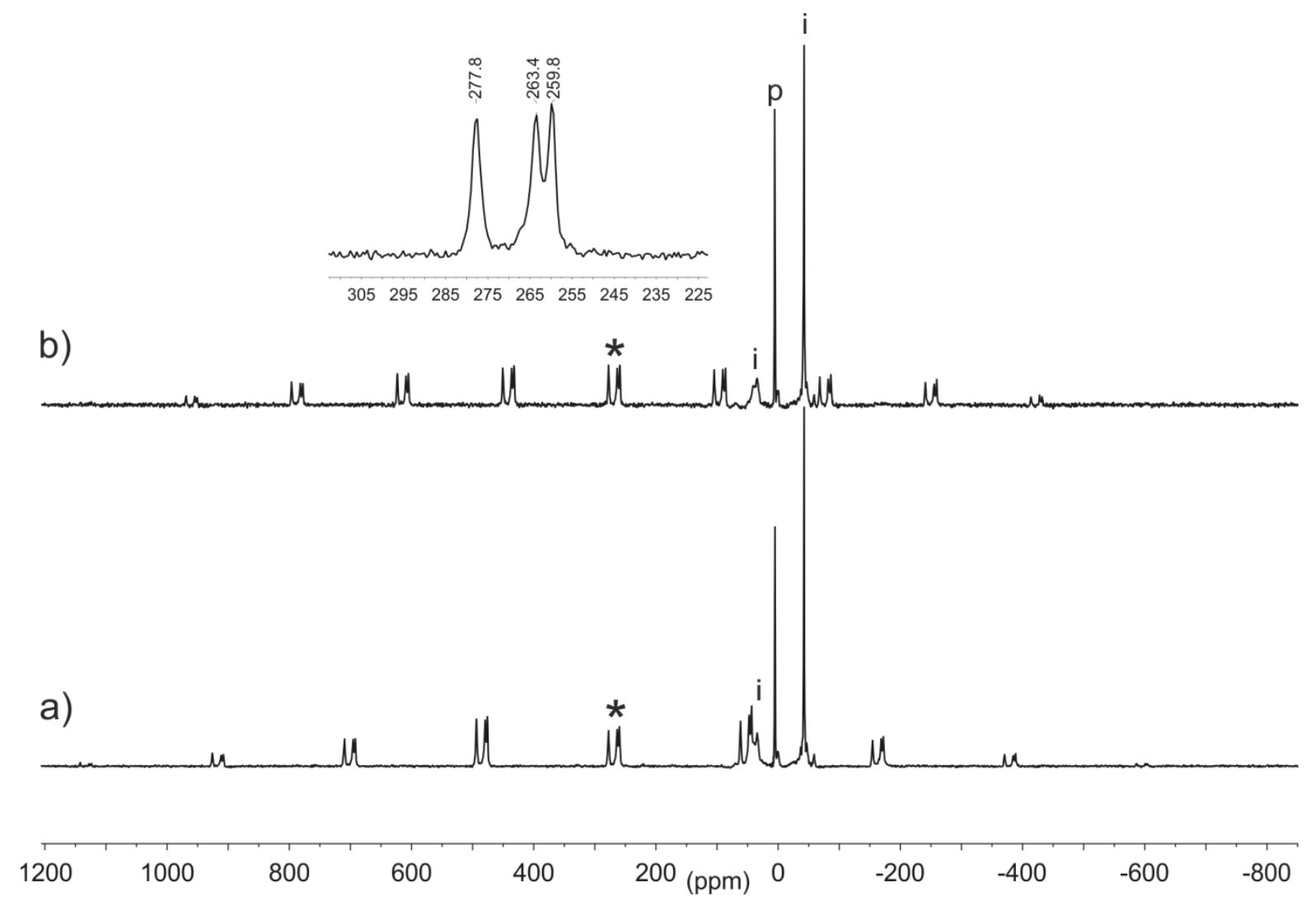

Figure S16: ${ }^{31} \mathrm{P}\left\{{ }^{1} \mathrm{H}\right\}$ CPMAS spectra of 3; a) spinning speed $35 \mathrm{kHz}$, b) spinning speed 28 $\mathrm{kHz}$, * indicates isotropic peak for $\mathrm{P} \equiv \mathrm{N}$ resonances (see inset expansion), $\mathrm{p}$ indicates $\mathrm{PMe}_{3}$ resonances, and i indicates impurity peaks. 


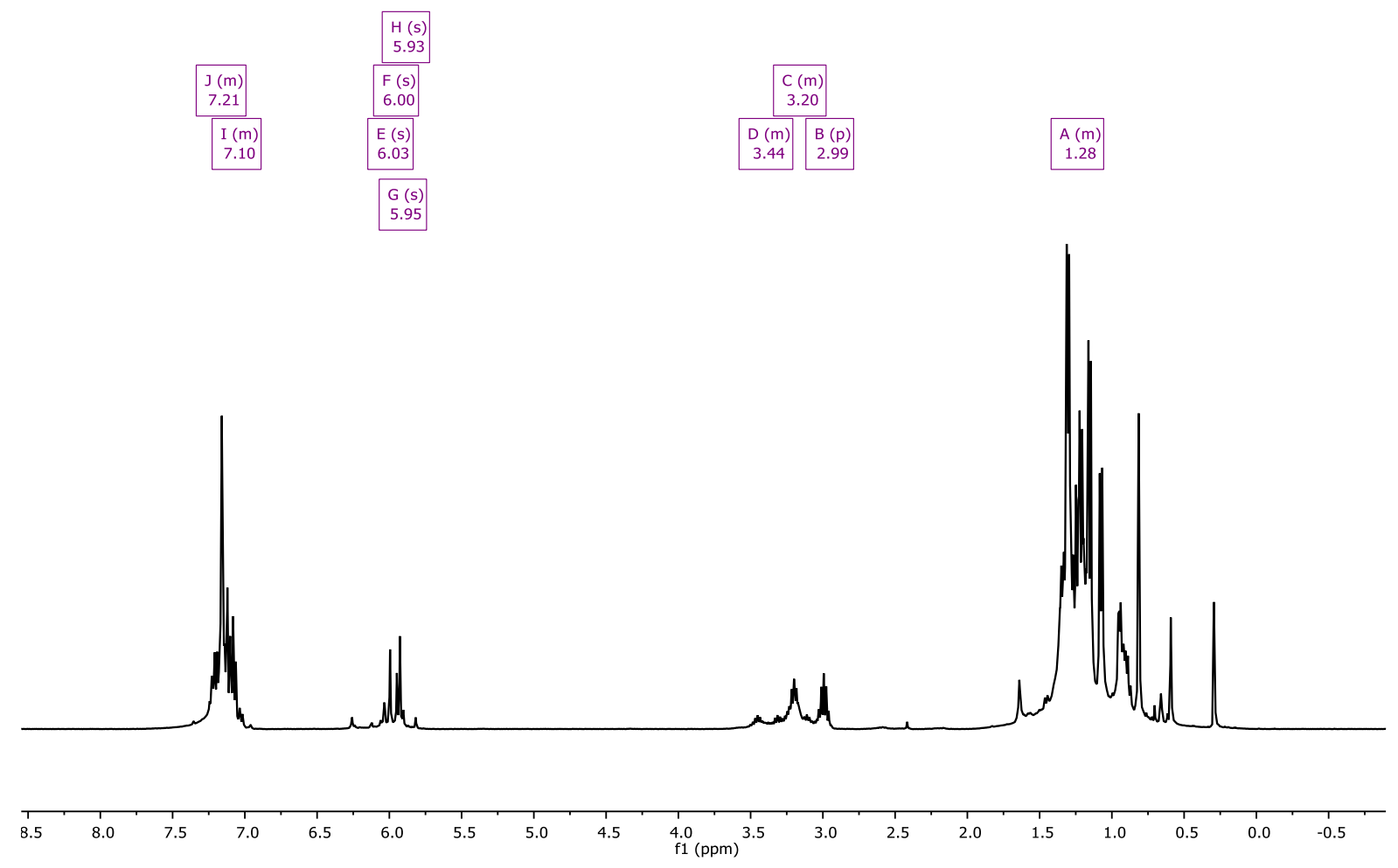

Figure S17: Room temperature ${ }^{1} \mathrm{H}$ spectrum of 4 in $\mathrm{C}_{6} \mathrm{D}_{6}$.

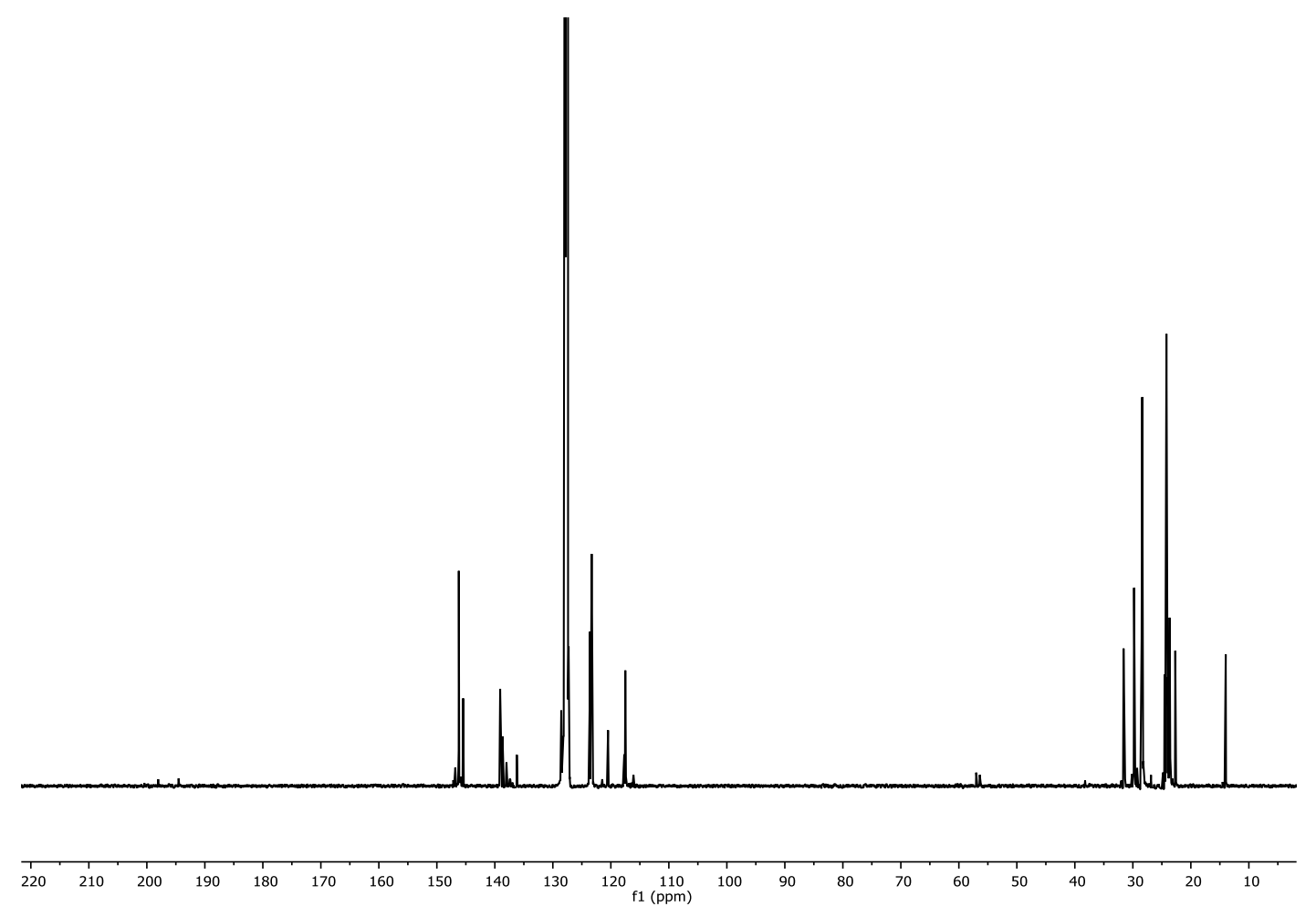

Figure S18: Room temperature ${ }^{13} \mathrm{C}\left\{{ }^{1} \mathrm{H}\right\}$ spectrum of $\mathbf{4}$ in $\mathrm{C}_{6} \mathrm{D}_{6}$. 


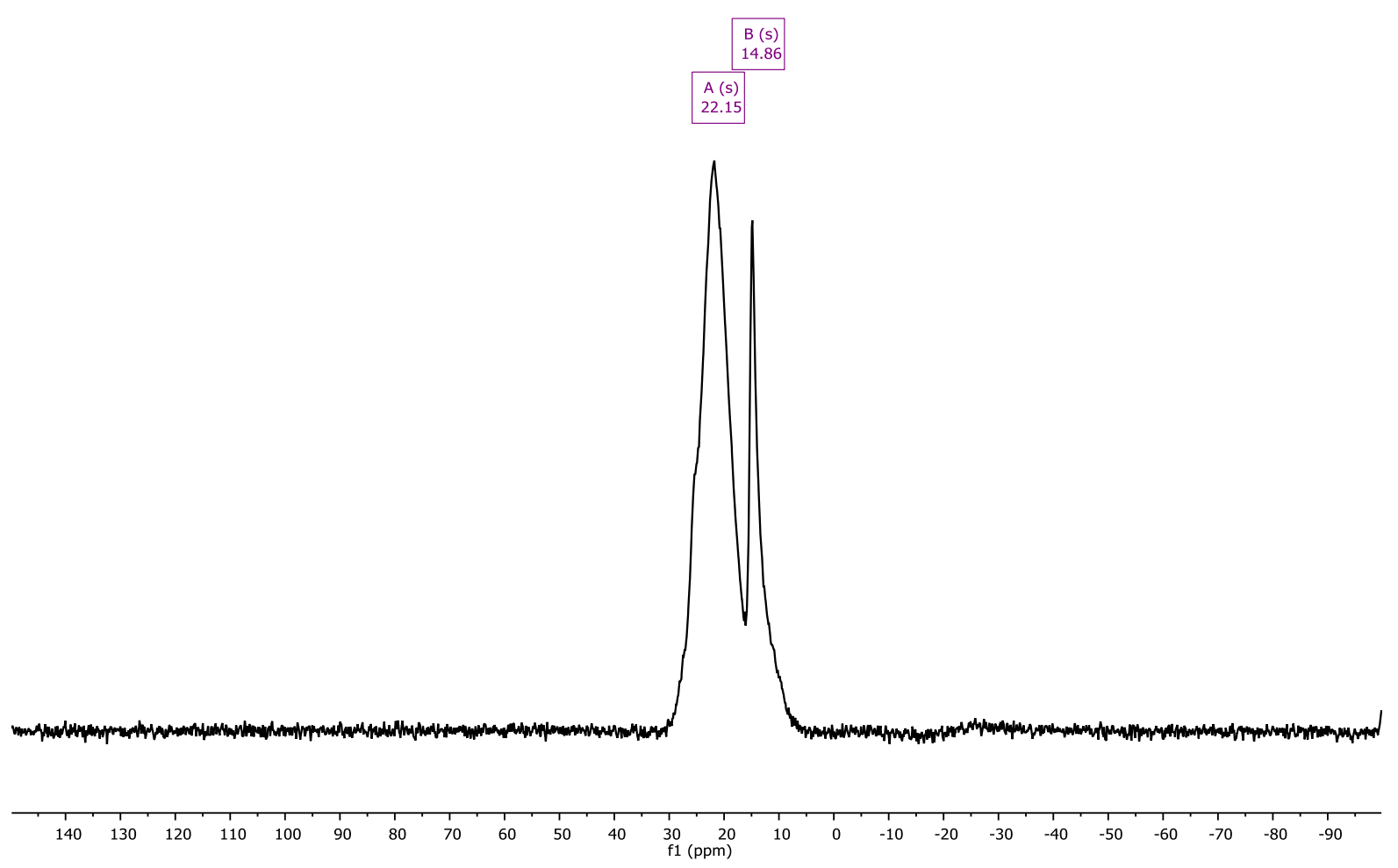

Figure S19: Room temperature ${ }^{11} \mathrm{~B}\left\{{ }^{1} \mathrm{H}\right\}$ spectrum of $\mathbf{4}$ in $\mathrm{C}_{6} \mathrm{D}_{6}$. 


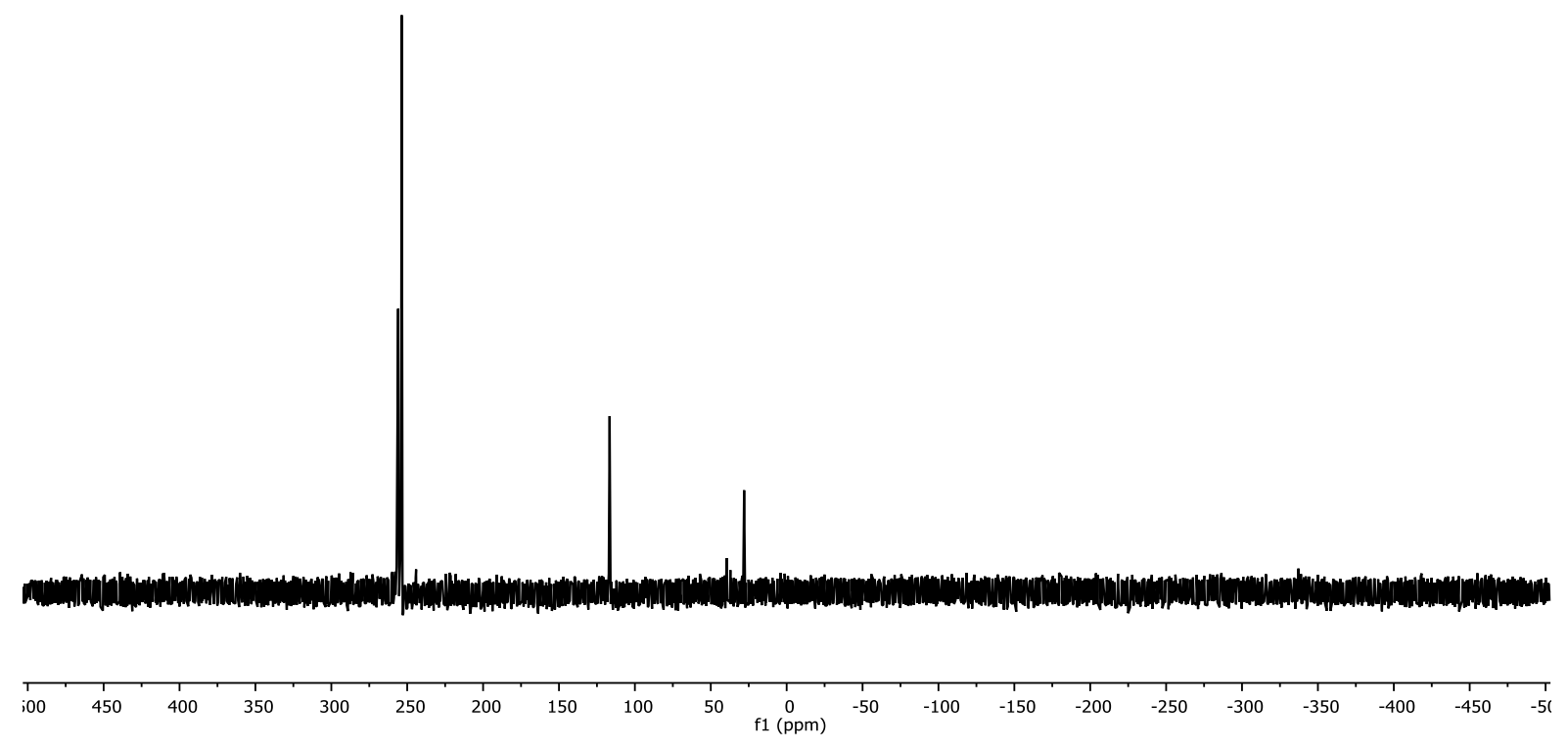

Figure S20: Room temperature ${ }^{31} \mathrm{P}\left\{{ }^{1} \mathrm{H}\right\}$ spectrum of $\mathbf{4}$ in $\mathrm{C}_{6} \mathrm{D}_{6}$.

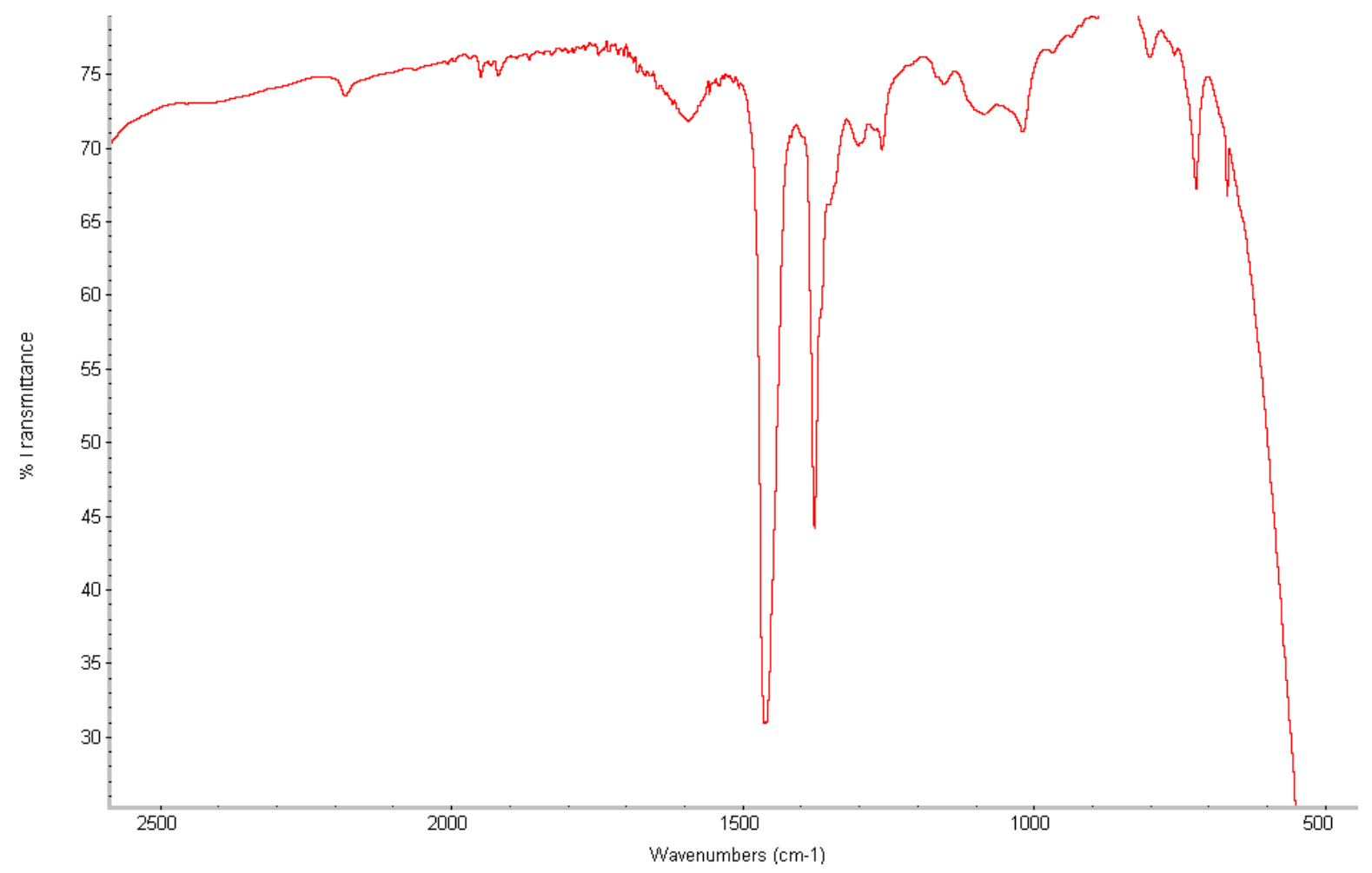

Figure S21: Solid state IR spectrum of 4. 


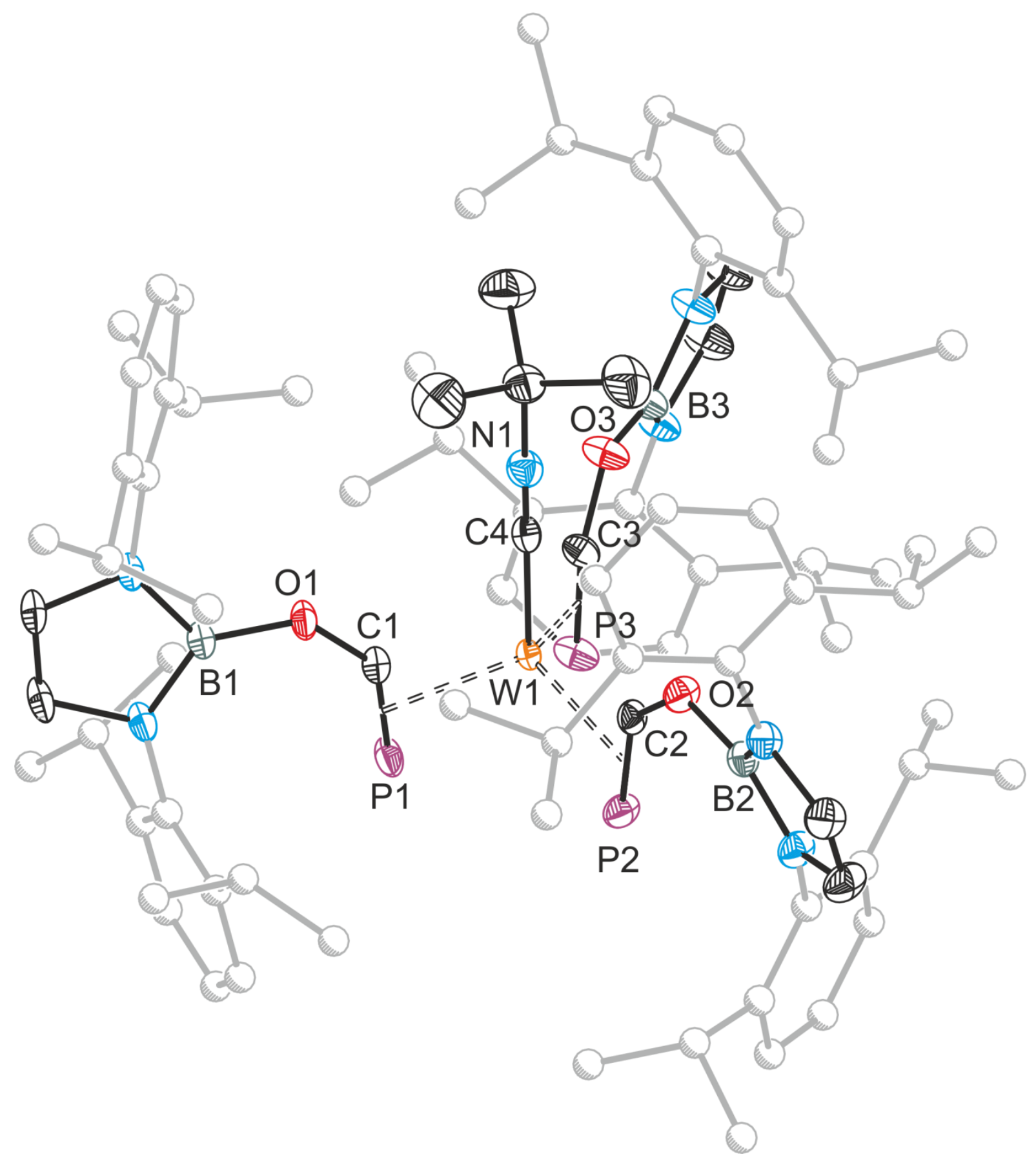

Figure S22. Molecular structure of 4a. Ellipsoids set at 50\% probability. Atoms of Dipp groups pictured as spheres of arbitrary radius. Select interatomic distances $[\AA]$ and angles $\left[{ }^{\circ}\right]$ : W1-P1 2.419(1), W1-P2 2.423(1), W1-P3 2.431(1), W1-C1 2.069(2), W1-C2 2.069(2), W1-C3 2.069(2), P1-C1 1.677(2), P2-C2 1.672(2), P3-C3 1.672(2), W1-C4 2.101(2) C4N1 1.148(3); W1-P1-C1 57.31(7), W1-P2-C2 57.22(7), W1-P3-C3 57.03(7), P1-C1-O1 139.94(15), P2-C2-O2 140.23(15), P3-C3-O3 140.47(16), W1-C4-N1 179.7(2). 


\section{Single crystal $X$-ray diffraction data}

Table S1. Selected X-ray data collection and refinement parameters for $\mathbf{2 a}, \mathbf{3 a}$ and $\mathbf{4 a} \cdot 0.5 \mathrm{hex}$.

\begin{tabular}{|c|c|c|c|}
\hline & $2 \mathbf{a}$ & $\mathbf{3 a}$ & 4a.0.5hex \\
\hline $\mathrm{FW}\left[\mathrm{g} \mathrm{mol}^{-1}\right]$ & 1550.93 & 1598.99 & 1649.13 \\
\hline Space group & $\operatorname{Pna}_{1}$ & Pna $_{1}$ & $P-1$ \\
\hline$a(\AA)$ & $19.763(4)$ & $20.0708(1)$ & $14.7117(1)$ \\
\hline$b(\AA)$ & $16.566(3)$ & $16.5566(1)$ & $22.5626(2)$ \\
\hline$c(\AA)$ & $25.827(5)$ & $26.1693(2)$ & $28.9916(3)$ \\
\hline$\gamma\left(\left(^{\circ}\right)\right.$ & 90 & 90 & $87.337(1)$ \\
\hline$V\left(\AA^{3}\right)$ & $8456(3)$ & $8696.17(10)$ & $8989.02(15)$ \\
\hline$Z$ & 4 & 4 & 4 \\
\hline Radiation, $\lambda(\AA)$ & $\mathrm{Cu} \mathrm{K} \alpha, 1.54184$ & $\mathrm{Cu} \mathrm{K} \alpha, 1.54184$ & $\mathrm{Cu} \mathrm{K \alpha}, 1.54184$ \\
\hline Temp (K) & $150(2)$ & $150(2)$ & $150(2)$ \\
\hline$\rho_{\text {calc }}\left(\mathrm{g} \mathrm{cm}^{-3}\right)$ & 1.218 & 1.221 & 1.219 \\
\hline $\mathrm{R}($ int $)$ & 0.0440 & 0.0456 & 0.0426 \\
\hline $\mathrm{R} 1 / \mathrm{wR} 2,{ }^{[\mathrm{a}]} \mathrm{I} \geq 2 \sigma \mathrm{I}(\%)$ & $5.76 / 15.25$ & $5.38 / 13.96$ & $2.80 / 6.81$ \\
\hline $\mathrm{R} 1 / \mathrm{wR} 2,{ }^{[\mathrm{a}]}$ all data $(\%)$ & $5.97 / 15.55$ & $6.03 / 14.76$ & $3.23 / 7.11$ \\
\hline GOF & 1.046 & 1.032 & 1.033 \\
\hline
\end{tabular}

2a, 0.0903 and 22.05 for $\mathbf{3 a}$, and 0.0326 and 8.97 for $\mathbf{4 a} \cdot 0.5$ hex. 
Table S2: Selected bond lengths for complexes $2 a, 3 a$ and $4 a$

\begin{tabular}{|c|c|c|c|c|}
\hline Bond or Angle & $2 \mathbf{a}$ & $\mathbf{3 a}$ & $4 a(1)$ & $4 a(2)$ \\
\hline W1-C* & $2.066(8)$ & $2.060(8)$ & $2.0689(19)$ & $2.0675(2)$ \\
\hline W1-P* & $2.433(2)$ & $2.431(2)$ & $2.4242(5)$ & $2.4340(5)$ \\
\hline $\mathbf{C}^{*}-\mathbf{P}^{*}$ & $1.672(9)$ & $1.687(9)$ & $1.673(2)$ & $1.671(2)$ \\
\hline W1-C4 & $2.050(6)$ & NA & $2.101(2)$ & $2.0935(19)$ \\
\hline O*-B* & $1.396(11)$ & $1.378(10)$ & $1.391(3)$ & $1.390(3)$ \\
\hline $\mathbf{C}^{*}-\mathbf{O}^{*}-\mathrm{B}^{*}$ & $127.7(8)$ & $130.0(7)$ & $126.52(17)$ & $126.61(17)$ \\
\hline $\mathrm{C}^{*}-\mathrm{W} 1-\mathrm{P}^{*}$ & $42.7(3)$ & $43.2(3)$ & $42.83(6)$ & $42.79(6)$ \\
\hline $\mathbf{P}^{*}-\mathbf{C}^{*}-\mathbf{W} 1$ & $80.5(4)$ & $80.3(3)$ & $79.99(8)$ & $80.06(8)$ \\
\hline $\mathrm{C}^{*}-\mathbf{P} *-\mathrm{W} 1$ & $56.9(3)$ & $56.7(3)$ & $57.19(7)$ & $57.15(7)$ \\
\hline $\mathbf{P}^{*}-\mathbf{C}^{*}-\mathbf{O}^{*}$ & $142.7(7)$ & $140.0(7)$ & $140.21(15)$ & $140.18(15)$ \\
\hline W1-C4-O4 & $178.7(8)$ & NA & NA & NA \\
\hline
\end{tabular}

*Average of all bond lengths/angles taken. Standard deviations were propagated according to reference [1] using the formula, where $v$ is the value and $\delta v$ is the standard deviation:

$$
\frac{\delta v}{v}=\sqrt{\left(\frac{\delta a}{a}\right)^{2}+\left(\frac{\delta b}{b}\right)^{2}}
$$




\section{Computational data}

Due to the steric requirement of the 2,6-diisopropylphenyl groups, it was not possible to construct and optimize isomers $\mathbf{c}$ and $\mathbf{d}$ for any of the molecules reported in this manuscript. Replacing these for phenyl groups resulted in the same issue. Further reduction to methyl allowed for optimisation of all but one isomer, with the complete set available for compound 3. The simplified model of $\mathbf{2 a}$ would not optimise due to a low barrier of rotation around the boryl groups which prevented convergence, a consequence of it being the least sterically encumbered isomer. We attempted to resolve this issue by using an ultrafine grid and adjusting the starting points of the atoms prior to optimisation, however in this case methyl rotation still prevented convergence.

Table S3: Summary table of optimised structures $\mathbf{2 a}^{\mathrm{Me}}-\mathbf{3} \mathbf{d}^{\mathrm{Me}} . \mathrm{W}\left(\eta^{2}-\mathrm{PCO}\left[\mathrm{B}^{\mathrm{Me}}\right]\right)_{3}(\mathrm{~L})$ $\left[\mathrm{B}^{\mathrm{Me}}\right] \mathrm{OCP}\left(\left[\mathrm{B}^{\mathrm{Me}}\right]=N, N^{\prime}\right.$-bis(methyl)-2,3-dihydro-1H-1,3,2-diazaboryl $) ; \mathrm{L}=\mathrm{CO}\left(\mathbf{2}^{\mathrm{Me}}\right), \mathrm{PMe}_{3}$ $\left(3^{\mathrm{Me}}\right)$.

\begin{tabular}{lllll}
\hline & E [a.u] & G [a.u.] & 31P Predicted & 31P Observed \\
\hline $\mathbf{2 a}$ & N.A. & N.A. & N.A. & 244.17 \\
$\mathbf{2 b}^{\mathrm{Me}}$ & -2418.007767 & -2417.658007 & $104.2,254.1$ & $109.3,244.6$ \\
$\mathbf{2} \mathbf{c}^{\mathrm{Me}}$ & -2418.00992936 & -2417.661325 & $242.6,105.8$ & N.A. \\
$\mathbf{2 d}^{\mathrm{Me}}$ & -2418.01410959 & -2417.662994 & 68.5 & N.A. \\
$\mathbf{3 a}^{\mathbf{M e}}$ & -2765.637663 & -2765.18289 & $33.4,275.5$ & $7.11,264.8$ \\
$\mathbf{3 b}^{\mathrm{Me}}$ & -2765.637242 & -2765.183192 & $21.8,100.8,278.3$ & $2.7,113.0,264.4$ \\
$\mathbf{3 c}^{\mathrm{Me}}$ & -2765.638641 & -2765.186075 & $10.4,99.1,265.4$ & N.A. \\
$\mathbf{3 d}^{\mathrm{Me}}$ & -2765.642425 & -2765.189262 & $-3.4,56.2$ & N.A. \\
\hline
\end{tabular}


Table S4: Summary of relative energies of isomers a-c.

\begin{tabular}{lllll}
\hline & $\mathbf{a}$ & $\mathbf{b}$ & $\mathbf{c}$ & $\mathbf{d}$ \\
\hline $\mathbf{E}(\mathbf{k J} / \mathbf{m o l})$ & N.A. & 0 & -5.677932555 & -16.65312642 \\
$\mathbf{R}=\mathbf{C O}$ & & & & \\
$\mathbf{G}(\mathbf{k J} / \mathbf{m o l})$ & N.A. & 0 & -8.711409 & -13.0933685 \\
$\mathbf{R}=\mathbf{C O}$ & & & & \\
$\mathbf{E}(\mathbf{k J} / \mathbf{m o l})$ & 0 & 1.106018 & -2.566111191 & -12.5013 \\
$\mathbf{R}=\mathbf{P M e} 3$ & & & & -16.729686 \\
$\mathbf{G}(\mathbf{k J} / \mathbf{m o l})$ & 0 & -0.7929 & -8.36222 & \\
$\mathbf{R}=\mathbf{P M e} 3$ & & & & \\
\hline
\end{tabular}

\section{References}

[1] I. G. Hughes, T. P. Hase, Measurements and their Uncertainties: A practical guide to modern error analysis, Oxford University Press, Oxford, UK, 2010. 